ressengruppen, können von Parlamenten zwar nicht unmittelbar beeinflusst werden; Steuerungsmöglichkeiten bestehen jedoch hinsichtlich der kommunikativen Leistungsfähigkeit von Parlamenten. Diese kann gezielt durch die Zahl und Qualität der Zugangspunkte für den Bürger, die Art der Mediennutzung, die Schaffung von Kapazitäten zur Verarbeitung und Bereitstellung von Informationen und die Ausgestaltung der institutionellen Regeln zur Herstellung von Öffentlichkeit und Transparenz gestaltet werden. Aus diesen Überlegungen folgt, dass die kommunikative Kapazität des Parlaments und damit dessen Responsivität durch das parlamentarische Petitionswesen als einem der Zugangspunkte zur Repräsentativversammlung zumindest in einem Teilbereich mit beeinflusst werden kann. Neben der institutionellen Ausgestaltung des Petitionsverfahrens (zum Beispiel Zugangsbedingungen, Anhörungsrechte) stellen Entscheidungen der Parlamentsakteure über die eingesetzte Medientechnologie (zum Beispiel Parlaments-TV, neue Medien) und die Kommunikationspraxis (zum Beispiel Informationsbereitstellung, Transparenz) mögliche „Stellschrauben" der kommunikativen Leistungsfähigkeit dar.

Inwieweit die jüngsten Reformen des Petitionswesens beim Bundestag einen Beitrag zu einem Demokratiewandel leisten oder lediglich eine "Scheinmodernisierung“ darstellen, lässt sich nun leichter erörtern. Aufgrund des für das politische Institutionensystem und dessen Machtkreisläufe insgesamt eher randständigen Petitionswesens ist ein grundlegender Demokratiewandel, ausgelöst durch Reformen in diesem Bereich, ohnehin nicht zu erwarten. Allerdings wurden durch die Bereitstellung eines neuen Einreichungskanals, die zaghafte Erhöhung der Verfahrenspublizität und die - gemessen an den technischen Möglichkeiten sehr zurückhaltende - Nutzung der neuen Medien die kommunikativen Kapazitäten des Petitionsausschusses verbessert. Dies ist wiederum mehr als eine bloße Scheinmodernisierung, die lediglich auf einen Imagegewinn durch den Einsatz neuer Medien setzt.

\title{
Öffentliche Petitionen beim Deutschen Bundestag. Ergebnisse einer Petentenbefragung
}

\author{
Ulrich Riehm und Matthias Trénel
}

Eine wissenschaftliche, empirisch ausgerichtete Petitionsforschung ist in Deutschland kaum entwickelt. Obwohl Petitionen eine der wesentlichen Möglichkeiten politischer Partizipation darstellen, werden sie in fast allen gängigen Standardbefragungen zur politischen Beteiligung so gut wie nicht aufgeführt. ${ }^{1}$ Erst seit jüngster Zeit gibt es Daten zum Ansehen des Petitionswesens in der Bevölkerung. ${ }^{2}$ Weitergehende Petentenbefragungen, unter anderem

1 Markus Steinbrecher, Politische Partizipation in Deutschland, Baden-Baden 2009, S. 314 ff. Steinbrecher stellt Fragebatterien zu den Verhaltensabsichten, Verhaltensmanifestationen und zur Effektivität politischer Partizipation aus unterschiedlichen Untersuchungen zusammen. Nur in einer von sechs Fragebatterien werden Petitionen beiläufig erwähnt.

2 Barbara Lippa / Herbert Kubicek / Stephan Bröchler, Bekanntheit und Ansehen des Petitionsausschusses des Deutschen Bundestages und Nutzung des Petitionsrechts in Deutschland, Berlin 
zu den Erwartungen an das Petitionsverfahren und zum realen Ablauf, sind aus Schottland bekannt. ${ }^{3}$ In Deutschland konnte eine Befragung aus dem Jahr 1968 ermittelt werden, in der das „Verfassungsbewusstsein“ von 676 Petenten aus den Jahren 1964 bis 1967 erhoben wurde. ${ }^{4}$ In der letzten Zeit hat bei Befragungen zur internetgestützten Partizipation das Thema Petitionen und E-Petitionen eine gewisse Beachtung gefunden. ${ }^{5}$

Die im Rahmen des Projekts „Öffentliche elektronische Petitionen und bürgerschaftliche Teilhabe" des Büros für Technikfolgen-Abschätzung beim Deutschen Bundestag (TAB ${ }^{6}$ durchgeführten Befragungen von Petenten liefern nun zum ersten Mal umfangreiche Daten zur Soziodemografie der Petenten, zu den mit Petitionen verbundenen Absichten sowie zum konkreten Petitionsprozess und seiner Bewertung. 7 Zum Projekt gehörte auch eine Evaluation des Modellversuchs „Öffentliche Petitionen“ des Bundestages. Diese beinhaltete - neben software-ergonomischen Untersuchungen, Analysen des herkömmlichen und neuen Petitionsverfahrens in der Bundestagsverwaltung, Befragungen von Abgeordneten des Petitionsausschusses und ihren Mitarbeitern - zwei schriftliche Befragungen von Petenten, die das bisherige Verfahren und jenen, die das neue, elektronische Verfahren nutzten.

Ein Gegenstand der Befragung waren die persönlichen Merkmale der Petenten. Beim Bundestag lagen bisher entweder nur anekdotische oder sehr begrenzte statistische Daten zur sozialen Zusammensetzung der Petenten vor. ${ }^{8}$ Die Befragungen des TAB können nun erstmals eine umfassendere und genauere Informationsbasis der Soziodemografie der Petenten liefern. Ergänzt wurden diese durch Daten zu Umfang und Art der Internetnutzung der Petenten sowie zum politischen Interesse und zur politischen Teilhabe. Man kann darüber Aufschluss gewinnen, ob die Hoffnung berechtigt ist, dass über den neuen „Petitionskanal“ Internet neue Bevölkerungsgruppen gewonnen werden können, und ob der „Partizipations-

2009. Auf einige Befragungen im Ausland (Österreich, Europa, Großbritannien) wird im Vorwort des Berichtes von Ulrich Riehm hingewiesen.

3 Christopher Carman, The Assessment of the Scottish Parliament's Public Petitions System 1999 - 2006, Edingburgh 2006; Christopher Carman, Modelling Petitioner Engagement with the Scottish Parliament's Petitions System: Procedural Fairness and Participatory Democracy, Glasgow 2007; Ipsos MORI / Christopher Carman, Engaging the Public in the Scottish Parliament's Petitions Process, Edinburgh 2009.

4 Peter Günther, Verfassungsrecht und Verfassungswirklichkeit des Petitionsrechts, Köln 1972. Günther bescheinigte den Petenten, dass sie überwiegend keine Nörgler und Querulanten seien, sondern im Vergleich zum Durchschnitt der Bevölkerung politisch interessierter und informierter sind und ein staatsbürgerliches Verhalten an den Tag legen. Demnach erscheint die Forderung nach sachgerechter Ausweitung der Kompetenzen des Petitionsausschusses zum Zwecke der besseren Erfüllung des subjektiv öffentlichen Rechtes auf sachliche Erledigung der Petitionen durch das ausgeprägte Verfassungsbewusstsein der Petenten legitim (S. 142).

5 Vgl. etwa Steffen Albrecht / Niels Kohlrausch / Herbert Kubicek / Barbara Lippa u.a., E-Partizipation - Elektronische Beteiligung von Bevölkerung und Wirtschaft am E-Government, Bremen 2008, S. 124 oder William Dutton / Ellen Helsper, The Internet in Britain: 2007, Oxford 2007, S. 72 f.

6 Siehe auch den Beitrag von Ralf Lindner und Ulrich Riehm in diesem Heft; Ulrich Riehm I Christopher Coenen / Ralf Lindner / Clemens Blümel, Bürgerbeteiligung durch E-Petitionen. Analysen von Kontinuität und Wandel im Petitionswesen, Berlin 2009.

7 „Wir haben erstmals Angaben darüber, wer sich besonders stark an uns wendet." Gabriele Lösekrug-Möller in der Bundestagsdebatte zum Tätigkeitsbericht des Petitionsausschusses 2006 am 20. September 2007, Plenarprotokoll 16/115, S. 11871.

8 Die Petitionsstatistik weist nur das Geschlecht der Petenten aus, vgl. etwa BR-Drs. 16/9500, S. 64. 
kanal“ Petitionen eher von politisch Interessierten und Aktiven genutzt wird oder auch von politikfernen Bevölkerungsgruppen.

Außerdem wurde nach den Inhalten der eingereichten Petitionen, den damit verfolgten Absichten und den Erwartungen an das Petitionsverfahren gefragt. Da die Befragung eng an die Einreichung einer bestimmten Petition gekoppelt war, konnten die konkreten Erfahrungen mit dem Verfahren ermittelt und die Zufriedenheit der Petenten mit der Behandlung ihrer Eingabe erfasst werden. Dies wurde separat auch für den Modellversuch „Öffentliche Petitionen" "9 und das darin etablierte Diskussionsforum erhoben. Außerdem konnten auch allgemeine Einschätzungen und Bewertungen abgegeben werden.

Insgesamt ist die folgende Auswertung und Darstellung auf zwei zentrale Fragen gerichtet: (1) Gelang es mit dem neuen internetgestützten Angebot öffentlicher Petitionen des Bundestages, andere Bevölkerungsgruppen zu erreichen als diejenigen, die bisher schon als Petenten in Erscheinung getreten sind? (2) Trifft die neue Art von Petitionen - öffentliche Petitionen im Internet mit Mitzeichnungs- und Diskussionsmöglichkeiten - auf einen Bedarf, und kann man auf Basis der Befragungsdaten etwas über das Nutzungspotenzial sagen?

\section{Methodisches Vorgehen}

Im Februar und März 2007 wurden eine Stichprobe der Einreicher traditioneller Petitionen und alle seit Beginn des Modellversuchs im September 2005 bekannten Einreicher öffentlicher Petitionen befragt (siehe Tabelle 1). ${ }^{10}$

\begin{tabular}{|c|c|c|}
\hline & $\begin{array}{l}\text { Einreicher herkömmlicher } \\
\text { Petitionen }\end{array}$ & $\begin{array}{l}\text { Einreicher öffentlicher } \\
\text { Petitionen }\end{array}$ \\
\hline Teilnehmer & $\begin{array}{l}\text { Einreicher herkömmlicher Petitionen, } \\
\text { die im Februar } 2007 \text { ihr Eingangs- } \\
\text { bestätigungsschreiben erhielten, dem } \\
\text { der Fragebogen beigelegt war. }\end{array}$ & $\begin{array}{l}\text { Alle Einreicher (zugelassener und } \\
\text { nichtzugelassener) öffentlicher Peti- } \\
\text { tionen, über deren Zulassung vor } \\
\text { dem } 1 . \text { Dezember } 2006 \text { entschieden } \\
\text { wurde. }^{\text {a }}\end{array}$ \\
\hline Befragungszeitraum & 1. Februar bis 5. März 2007 & 5. März bis 16. März 2007 \\
\hline verschickte Fragebögen & 1.000 & 698 \\
\hline Rücklauf & $571(57 \%)$ & $350(50 \%)$ \\
\hline Methode & \multicolumn{2}{|c|}{ schriftliche, postalische, standardisierte Befragung } \\
\hline \multicolumn{3}{|c|}{$\begin{array}{l}\text { a Zum Erhebungszeitpunkt war bei allen öffentlichen Petitionen der Befragten die sechswöchige Mit- } \\
\text { zeichnungs- und Kommentierungsphase im Internet bereits beendet. Davon abgesehen war das Bear- } \\
\text { beitungsstadium der öffentlichen Petition sehr unterschiedlich: Bei einigen lag die Einreichung bereits } \\
\text { über ein Jahr zurück, und hiervon waren einige Petitionen bereits abgeschlossen, bei anderen lag die } \\
\text { Einreichung der Petition erst drei Monate zurück. } \\
\text { Quelle: Eigene Darstellung. }\end{array}$} \\
\hline
\end{tabular}

9 Ulrich Riehm / Christopher Coenen / RalfLindner / Clemens Blümel, a.a.O. (Fn. 6), S. 207 - 238.

10 Die Befragungen wurden im Auftrag des Bundestages im Rahmen des Projekts „Öffentliche elektronische Petitionen und bürgerschaftliche Teilhabe" des Büros für Technikfolgen-Abschätzung beim Deutschen Bundestag (TAB) durch Zebralog e.V., Berlin, durchgeführt. 


\section{Ergebnisse}

\subsection{Neue Petentengruppen durch öffentliche Petitionen?}

Die Richtlinie für die Behandlung von öffentlichen Petitionen des Deutschen Bundestages $^{11}$ nennt als Ziele für öffentliche Petitionen, dass möglichst viele Petenten ihr Anliegen vorstellen können und ein möglichst breites Spektrum an Themen öffentlich gemacht werden kann. Außerdem versteht sich der Petitionsausschuss als Seismograf für Stimmungen in der Bevölkerung. ${ }^{12}$ Damit ist die Frage nach der Repräsentativität der Petenten gestellt.

Die Ergebnisse der Befragungen zeigen, dass sich die Einreicher herkömmlicher wie öffentlicher Petitionen erheblich vom Durchschnitt der Bevölkerung unterscheiden (siehe Tabelle 2): Unter den Petenten sind überdurchschnittlich viele Männer ${ }^{13}$, ihr Ausbildungsniveau ist höher, und bei ihnen besteht eine amtlich festgestellte Behinderung deutlich häufiger.

Die beiden hier betrachteten Petentengruppen, die Einreicher traditioneller wie öffentlicher Petitionen, weisen aber auch markante Unterschiede untereinander auf: Die Einreicher öffentlicher Petitionen sind deutlich jünger (ihr Anteil an der Altersgruppe der 20- bis 39-Jährigen beträgt 32,7 Prozent und entspricht damit in etwa dem Bevölkerungsdurchschnitt, derjenige der traditionellen Petenten liegt bei 13,2 Prozent); ihr Ausbildungsniveau ist noch höher als das der traditionellen Petenten (Anteil mit Hochschulabschluss 43,7 vs. 31,7 Prozent); unter ihnen ist ein hoher Anteil an Selbstständigen (19,5 vs. 8,6 Prozent); sie sind zu einem größeren Anteil Auszubildende, Schüler, Studenten oder Zivil- beziehungsweise Grundwehrdienstleistende (9,3 vs. 4,6 Prozent); ihr Anteil unter den Rentnern ist dagegen deutlich geringer (14,0 vs. 39,8 Prozent).

So scheint es zwar gelungen zu sein, neue Bevölkerungsschichten durch das Angebot öffentlicher Petitionen zu erreichen, aber nicht in dem vielleicht erhofften Sinn. Denn Frauen, Petenten ohne Hochschulabschluss, auch Erwerbslose und dauerhaft Arbeitsunfähige und Behinderte konnten ihren Anteil unter den Einreichern öffentlicher Petitionen nicht erhöhen, sondern sind gegenüber den Einreichern traditioneller Petitionen weiter zurückgefallen. Dem Ziel, die Petenten sollten einem repräsentativen Querschnitt der Bevölkerung entsprechen ${ }^{14}$, ist man über öffentliche Petitionen so höchstens einen kleinen Schritt näher gekommen.

11 Deutscher Bundestag, Richtlinie für die Behandlung von öffentlichen Petitionen (öP) gemäß Ziffer 7.1 (4) der Verfahrensgrundsätze, https://epetitionen.bundestag.de/index.php?\&action =policy (Abruf am 11. Juni 2009).

12 Deutscher Bundestag, Stichwort Petitionen, Von der Bitte zum Bürgerrecht, Berlin 2007, S. 41.

13 Die Angaben zum Geschlecht stimmen im Übrigen gut mit den diesbezüglichen Angaben in der Petitionsstatistik des Deutschen Bundestages überein. Danach waren 26,4 Prozent der Petitionsneueingänge natürlicher Personen im Jahr 2007 von Frauen, 73,6 Prozent von Männern. Die Männerdominanz weist seit dem Beginn der Aufzeichnungen 1977 nur geringe Schwankungen auf und wurde in den letzten 30 Jahren nicht reduziert. Der Männeranteil lag für den Zeitraum 14. Dezember 1976 bis 31. Dezember 1978 bei 69,0 Prozent; der niedrigste Anteil wird für 1992 mit 62,3 Prozent ausgewiesen. Vgl. BT-Drs. 16/9500, S. 64; BT-Drs. 8/3036, S. 48; BT-Drs. 12/4961, S. 91.

14 Die Abgeordnete Gabriele Lösekrug-Möller wies in der Plenardebatte des Bundestages über den Tätigkeitsbericht des Petitionsausschusses darauf hin, dass der Regelpetent - männlich, älter als 40 Jahre, Hochschulabschluss - beim Petitionsausschuss jederzeit willkommen sei, sie sich aber wünsche, dass sich auch verstärkt Migranten, Frauen und Jüngere an den Bundestag mit ihren Anliegen wenden würden, Stenografischer Bericht, 115. Sitzung, Berlin, 20. September 2007, Plenarprotokoll 16/115, S. $11871-11872$. 


\begin{tabular}{|c|c|c|c|}
\hline & $\begin{array}{c}\text { Einreicher traditio- } \\
\text { neller Petitionen }\end{array}$ & $\begin{array}{c}\text { Einreicher öffentli- } \\
\text { cher Petitionen }\end{array}$ & $\begin{array}{c}\text { Bevölkerung } \\
\text { (Mikrozensus) }\end{array}$ \\
\hline Geschlecht & $\mathrm{n}=564$ & $\mathrm{n}=347$ & \\
\hline männlich & 73,9 & 75,8 & 48,9 \\
\hline weiblich & 26,1 & 24,2 & 51,1 \\
\hline Altersgruppen & $\mathrm{n}=560$ & $\mathrm{n}=342$ & $\begin{array}{l}\text { Bevölkerung } \\
\text { ab } 20 \text { Jahre }\end{array}$ \\
\hline 20 bis 39 Jahre & 13,2 & 32,7 & 33,2 \\
\hline 40 bis 59 Jahre & 40,4 & 52,0 & 35,5 \\
\hline 60 bis 99 Jahre & 46,4 & 15,2 & 31,3 \\
\hline Ausbildung & $\mathrm{n}=571$ & $\mathrm{n}=350$ & $\begin{array}{l}\text { Bevölkerung } \\
\text { ab } 15 \text { Jahre }\end{array}$ \\
\hline $\begin{array}{l}\text { noch in Ausbildung / Studium bezie- } \\
\text { hungsweise ohne beruflichen Abschluss }\end{array}$ & 8,9 & 10,6 & 30,6 \\
\hline $\begin{array}{l}\text { Abschluss Lehrausbildung, Berufsfach- } \\
\text { schule oder Anlernausbildung }\end{array}$ & 32,6 & 28,6 & 48,8 \\
\hline $\begin{array}{l}\text { Meister- / Technikerausbildung oder } \\
\text { gleichwertiger Fachschulabschluss }\end{array}$ & 19,0 & 11,1 & 6,9 \\
\hline $\begin{array}{l}\text { Hochschulabschluss (Fachhochschule } \\
\text { und Universität) }\end{array}$ & 31,7 & 43,7 & 11,8 \\
\hline anderer Abschluss & 7,9 & 6,0 & 0,6 \\
\hline Erwerbstätigkeit & $\mathrm{n}=548$ & $\mathrm{n}=344$ & \\
\hline Angestellter, Arbeiter, Beamter & 25,5 & 45,3 & 39,8 \\
\hline Selbstständiger & 8,6 & 19,5 & 6,4 \\
\hline Ruhestand, Vorruhestand & 39,8 & 14,0 & 28,3 \\
\hline dauerhaft arbeitsunfähig & 6,9 & 1,5 & 3,5 \\
\hline arbeitslos & 12,0 & 8,7 & 7,1 \\
\hline Hausfrau, Hausmann & 2,6 & 1,7 & 9,2 \\
\hline $\begin{array}{l}\text { Auszubildender, Schüler, Student, } \\
\text { Grundwehr- / Zivildienstleistender }\end{array}$ & 4,6 & 9,3 & 5,8 \\
\hline amtlich festgestellte Behinderung & $\mathrm{n}=571$ & $\begin{array}{r}\mathrm{n}=346 \\
15,6\end{array}$ & 8,0 \\
\hline
\end{tabular}

\subsection{Potenziale für öffentliche Petitionen}

Ein weiteres Ziel des Modellversuchs bestand darin, mit der Einführung der Eingabemöglichkeit über E-Mail und das Web auf die zunehmende Nutzung des Internets in der Bevölkerung zu reagieren. Diese Modernisierung des Petitionswesens war mit der Hoffnung verbunden, über diesen Weg insbesondere jüngere Bevölkerungsgruppen zu erreichen, die bisher unter den Petenten stark unterrepräsentiert waren. Diese Erwartung wurde - wie 
oben gezeigt - in einem gewissen Maße erfüllt. Zu fragen ist, ob das Potenzial an Einreichern öffentlicher Petitionen damit schon ausgeschöpft wurde. Eine erste Antwort kann man gewinnen, wenn man die Internetaffinität der Einreicher traditioneller Petitionen betrachtet. Reichen diese keine öffentlichen Petitionen ein, weil dazu die Nutzung des Internets vorausgesetzt wird und sie dazu generell keinen Zugang haben, oder sind es andere Gründe, die sie an der Einreichung öffentlicher Petitionen hindern?

Wie Tabelle 3 zeigt, kann der fehlende Internetzugang nicht die Hauptursache sein, denn die Einreicher herkömmlicher Petitionen liegen mit einem Anteil an Internetnutzern von 67,3 Prozent über dem Bevölkerungsdurchschnitt von 63 Prozent. ${ }^{15}$ Auch in Bezug auf einzelne Nutzungsformen wie E-Mail, Suchmaschinen oder Homebanking liegt bei den traditionellen Petenten das Nutzungsniveau höher als beim Bevölkerungsdurchschnitt. Nur unterhaltende Internetnutzungsformen (wie Computerspiele, Chatten) sind bei dieser Gruppe im Vergleich zur Gesamtbevölkerung seltener festzustellen, was vermutlich weitgehend mit dem höheren Altersschnitt der Einreicher herkömmlicher Petitionen erklärt werden kann.

\begin{tabular}{|c|c|c|c|}
\hline \multicolumn{4}{|l|}{ Tabelle 3: Internetnutzung (in Prozent) } \\
\hline & $\begin{array}{l}\text { Einreicher } \\
\text { traditioneller } \\
\text { Petitionen }\end{array}$ & $\begin{array}{l}\text { Einreicher } \\
\text { offentlicher } \\
\text { Petitionen }\end{array}$ & $\begin{array}{c}\text { Gesamt- } \\
\text { bevölkerung }\end{array}$ \\
\hline Internetnutzung (auch gelegentlich, beliebiger Ort) & 67,3 & 100,0 & 63 \\
\hline Seit wann nutzen Sie das Internet? (Mittelwert Jahre) & 7,4 & 9,1 & 7 \\
\hline \multicolumn{4}{|l|}{ Nutzen Sie das Internet für ... ${ }^{2}$} \\
\hline E-Mail & 86,3 & 95,9 & 79 \\
\hline Suchmaschinen & 80,3 & 93,8 & 76 \\
\hline Homebanking & 47,9 & 55,5 & 34 \\
\hline Gesprächsforen, Chatten & 16,6 & 29,9 & 20 \\
\hline Computer-/Onlinespiele & 3,1 & 6,2 & 10 \\
\hline \multicolumn{4}{|c|}{$\begin{array}{l}1 \text { Die Vergleichszahlen zur Gesamtbevölkerung ab } 14 \text { Jahren entstammen der ARD / ZDF-Online- } \\
\text { Studie } 2007 . \\
2 \text { Prozentuiert wird im Folgenden auf die Internetnutzer unter den Petenten beziehungsweise in der } \\
\text { Bevölkerung ab } 14 \text { Jahren. } \\
\text { Quelle: Eigene Erhebung 2007; Birgit von Eimeren / Beate Frees, a.a.O. (Fn.15). }\end{array}$} \\
\hline
\end{tabular}

Um die öffentliche Petition als Möglichkeit für die Einreichung eines Anliegens in Betracht ziehen zu können, muss man nicht nur Internetnutzer sein, sondern auch von dieser Möglichkeit wissen. Danach gefragt, antworteten nur 16,9 Prozent der Einreicher herkömmlicher Petitionen, dass sie diese Form der Einreichung kannten, was zum großen Teil erklärt, warum davon nicht häufiger Gebrauch gemacht wurde. Allerdings nutzten die meisten

15 Die Frageformulierung lautete: „Nutzen Sie selbst zumindest gelegentlich irgendwelche Onlinedienste beziehungsweise das Internet, egal ob zu Hause, am Arbeitsplatz, an der Universität, an der Schule oder unterwegs?" Diese Frageformulierung lehnt sich an den Fragebogen der ARD / ZDF-Online-Studie an, deren Daten zu Vergleichszwecken für die Internetnutzung der Bevölkerung herangezogen werden. Vgl. Birgit von Eimeren / Beate Frees, Internetnutzung zwischen Pragmatismus und YouTube-Euphorie, in: Media Perspektiven, 37. Jg. (2007), H. 8, S. $362-378$. 
dieser Nichtinformierten (82,6 Prozent) das Internet, wären also prinzipiell in der Lage gewesen, eine öffentliche Petition einzureichen.

Grundsätzlich fanden es 70,4 Prozent der befragten Einreicher traditioneller Petitionen sehr oder eher interessant, die eigene Petition über ein Onlineformular dem Bundestag zuzuleiten, was ebenfalls auf ein noch großes ungenutztes Potenzial für elektronische und öffentliche Petitionen schließen lässt. Tatsächlich hat jeder Vierte der befragten Einreicher herkömmlicher Petitionen das Internet zur Übermittlung seiner Petition an den Bundestag genutzt (20,7 Prozent per Online-Formular, 4,8 Prozent per E-Mail). ${ }^{16}$

\subsection{Mitsprache politikferner Bevölkerungsgruppen?}

Petitionen gelten im Vergleich zu anderen Möglichkeiten der politischen Partizipation als eine besonders einfache, niedrigschwellige Beteiligungsform. ${ }^{17}$ Es ist deshalb zu vermuten, dass Petitionen auch und in besonderem Maße von denjenigen Bevölkerungsgruppen genutzt werden, die von den sonstigen, höherschwelligen Formen politischer Beteiligung nicht so stark Gebrauch machen. Die Befragung zeigt allerdings ein anderes Ergebnis.

Generell ist das Niveau politischer Aktivität (Mitgliedschaft in Parteien und Verbänden, Teilnahme an Demonstrationen, Unterschriftensammlungen und sonstigen Petitionen, Kontakt mit Medien, Politikern oder Behörden) bei beiden Petentengruppen höher als beim Durchschnitt der Gesamtbevölkerung. Bemerkenswert ist allerdings, dass im Vergleich der beiden Petentengruppen die Einreicher öffentlicher Petitionen bei den angeführten Indikatoren - mit einer Ausnahme - überall eine höhere Beteiligungsquote aufweisen. Besonders augenfällig ist dies etwa bei der Teilnahme an einer Unterschriftensammlung mit 94,9 zu 80,9 Prozent, bei der Kontaktaufnahme zu Politik oder Verwaltung mit 70,8 zu 40,5 Prozent oder der Teilnahme an einer Demonstration mit 59,3 zu 47,8 Prozent.

Die erwartete „Empowerment-Wirkung“ durch öffentliche Petitionen tritt also nicht ein. Eher ist ein gegenteiliger Effekt zu erkennen, der auch an anderer Stelle schon festgestellt wurde. ${ }^{18}$ Gerade die ohnehin politisch aktiven Bevölkerungsteile werden durch die

16 Man muss dazu wissen, dass für die Einreicher öffentlicher Petitionen der Einreichungsweg vorgeschrieben ist - im Rahmen des Modellversuchs 2005 bis 2007 war dies ein digital auszufüllendes Formular im PDF-Dateiformat, das als Attachement per E-Mail an den Petitionsausschuss geschickt werden musste. Die Einreicher herkömmlicher Petitionen können dagegen verschiedene postalische oder elektronische Kanäle nutzen. Seit Oktober 2008 werden öffentliche Petitionen an den Deutschen Bundestag über ein Webformular eingereicht. Traditionelle Petitionen können weiterhin postalisch, über ein Webformular oder als Attachment einer E-Mail an den Bundestag übermittelt werden. Nach den nicht veröffentlichten Angaben des Deutschen Bundestages lag der Anteil der über das Onlineformular eingereichten traditionellen Petitionen im Jahr 2006 bei 12,6 Prozent und im Jahr 2007 bei 13,2 Prozent. Das sind deutlich weniger, als die Befragungsergebnisse für die Neueinreichungen im Februar 2007 (20,7 Prozent) ergeben haben. Dies mag mit einer stärkeren Antwortbereitschaft der Internetnutzer unter den Einreichern traditioneller Petitionen zu tun haben.

17 Vgl. RalfLinder / Ulrich Riehm, a.a.O. (Fn. 6).

18 Tobias Escher, Wi(e)der die üblichen Verdächtigen? Politische Beteiligung über das Internet. Eine empirische Untersuchung von bürgerinitiierten Online-Kontakten zu Abgeordneten über die Webseite WriteToThem.com in Großbritannien, in: Martin Emmer / Jens Wolling / Markus Seifert, Politik 2.0, in Vorbereitung. Allerdings ist der beobachtete Effekt bei Escher ein anderer als 


\begin{tabular}{l}
\hline \multicolumn{3}{|c|}{ Tabelle 4: Mitgliedschaften in politischen und sozialen Organisationen, Teilnahme an } \\
Demonstrationen (in Prozent)
\end{tabular}

öffentlichen Petitionen besonders gut erreicht. Statt eines Ausgleichs kommt es zu einer Verstärkung. Allerdings muss berücksichtigt werden, dass zum Untersuchungszeitpunkt die Möglichkeit zur Einreichung öffentlicher Petitionen der breiten Bevölkerung wenig bekannt war (siehe oben), und nur solche Personen davon erfahren konnten, die im stärkeren Maße politische Informationen im Internet rezipieren.

In den Diskussionen zur Einführung des Modellversuchs spielte das Argument des Missbrauchs der öffentlichen Petitionen durch Organisationen eine gewisse Rolle. Nun ist das Grundrecht auf Petitionen ausdrücklich nicht auf Einzelpersonen eingeschränkt, aber im Verständnis eines Teils der Abgeordneten sollte das Petitionsrecht in erster Linie dem einzelnen Bürger zugutekommen, da Organisationen über andere Mittel verfügen, sich in der Politik Gehör zu verschaffen. ${ }^{19}$ Aus Tabelle 4 ergibt sich ein minimaler Organisationsgrad der Einreicher öffentlicher Petitionen von 32,2 und ein maximaler von 50,6 Prozent. ${ }^{20}$ Diese Werte liegen bei den traditionellen Petenten mit minimal 26,5 und maximal 39,5 Prozent etwas niedriger. Dem entspricht, dass bei den Einreichern öffentlicher Petitionen mit 34,5 Prozent ein höherer Anteil der konkret eingereichten Petitionen in einem mehr oder weniger losen Bezug zu einer politischen Organisation steht als bei den traditionellen Petenten (21,9 Prozent). Für beide Gruppen ist jedoch der Anteil an Petitionen, die in einem direkten Bezug zu einer Organisation stehen („Petition entstand im Rahmen dieser

im vorliegenden Fall. Escher berichtet davon, dass durch politische Internetangebote sozial privilegierte, aber eher politisch inaktive Bevölkerungsteile mobilisiert werden konnten. Bei den Petenten ist es so, dass zwar neue (jüngere) Bevölkerungsgruppen durch das Internetangebot erreicht wurden, im überdurchschnittlichen Maße aber solche, die schon besonders politisch aktiv waren.

19 Vgl. Stephan Löwenstein, Eine Bühne für Masseneingaben, SPD und Grüne wollen das Petitionsrecht ändern, in: Frankfurter Allgemeine Zeitung vom 28. Dezember 2004, S. 4.

20 Bei der Berechnung des Minimalwertes wird angenommen, dass jedes Mitglied einer Gewerkschaft oder Berufsorganisation auch Mitglied einer Partei ist; bei der Berechnung des Maximalwertes wird angenommen, dass kein Mitglied einer Gewerkschaft oder eines Berufsverbandes auch Mitglied einer Partei ist. 
Organisation(en)“), mit 8,0 bei den traditionellen Petenten beziehungsweise 11,3 Prozent bei den Einreichern öffentlicher Petitionen relativ gering. Die Befürchtung also, öffentliche Petitionen würden massiv durch Organisationen in Anspruch genommen, wird von den vorliegenden Daten nicht gestützt.

Tabelle 5 enthält die Ergebnisse zur Nutzung weiterer Möglichkeiten der politischen Beteiligung, und zwar solche, die prinzipiell auch im Internet durchgeführt werden können. Wieder zeigt sich, dass die Petenten generell ein höheres politisches Aktivitätsniveau aufweisen als der Durchschnitt der Bevölkerung, und dass sich die Einreicher öffentlicher Petitionen (in drei von vier Merkmalen) häufiger politisch betätigen als die Einreicher traditioneller Petitionen.

Mehr als die Hälfte beider Gruppen geben an, dass sie - abgesehen von der aktuellen Petition an den Bundestag - bereits anderweitig Petitionen an Parlamente, Regierungsstellen oder Behörden gerichtet haben. Dies deutet darauf hin, dass das Instrument Petition, wenn es einmal von Bürgern entdeckt und genutzt wurde, gern erneut in Anspruch genommen wird. Diese Mehrfachnutzung politischer Beteiligungsformen wird in der Literatur oft negativ konnotiert und mit dem Terminus einer Nutzung durch die „üblichen Verdächtigen "versehen. ${ }^{21}$ Eine andere Interpretation könnte aber auch dahingehend formuliert werden, dass von dieser Personengruppe die Petition als nützliche Form des persönlichen Interessen- und Rechtsschutzes sowie als Möglichkeit politischer Partizipation erfahren wurde, und deshalb wiederholt eingesetzt wird.

\begin{tabular}{|c|c|c|c|c|c|}
\hline & \multicolumn{2}{|c|}{$\begin{array}{l}\text { Einreicher traditioneller } \\
\text { Petitionen }\end{array}$} & \multicolumn{2}{|c|}{$\begin{array}{l}\text { Einreicher öffentlicher } \\
\text { Petitionen }\end{array}$} & \multirow{2}{*}{$\begin{array}{l}\text { Gesamt- } \\
\text { bevölke- } \\
\text { rung }^{1}\end{array}$} \\
\hline & $\begin{array}{l}\text { habe ich } \\
\text { schon mal } \\
\text { getan }\end{array}$ & $\begin{array}{l}\text { wenn getan, } \\
\text { auch über } \\
\text { das Internet }\end{array}$ & $\begin{array}{l}\text { habe ich } \\
\text { schon mal } \\
\text { getan }\end{array}$ & $\begin{array}{l}\text { wenn getan, } \\
\text { auch über } \\
\text { das Internet }\end{array}$ & \\
\hline $\begin{array}{l}\text { an einer Unterschriftensamm- } \\
\text { lung teilgenommen } \\
(\mathrm{n}=535,302,332,285)\end{array}$ & 80,9 & 26,5 & 94,9 & 66,3 & 54,6 \\
\hline $\begin{array}{l}\text { eine Petition, abgesehen von } \\
\text { der aktuellen, eingereicht } \\
(\mathrm{n}=536,202,326,164)\end{array}$ & 54,3 & 28,7 & 54,0 & 53,7 & - \\
\hline $\begin{array}{l}\text { mit Medien Kontakt } \\
\text { aufgenommen } \\
(\mathrm{n}=536,230,325,219)\end{array}$ & 40,5 & 51,3 & 70,8 & 79,5 & - \\
\hline $\begin{array}{l}\text { mit Politik oder Verwaltung } \\
\text { Kontakt aufgenommen } \\
(\mathrm{n}=539,247,326,218)\end{array}$ & 71,1 & 36,8 & 73,3 & 69,3 & 20,9 \\
\hline \multicolumn{6}{|c|}{$\begin{array}{l}1 \text { Die Vergleichszahlen zur Gesamtbevölkerung entstammen dem Allbus 2006. Mehrfachantworten } \\
\text { möglich. } \\
\text { Quelle: Eigene Erhebungen 2007; Allbus, a.a.O. (Tabelle 4). }\end{array}$} \\
\hline
\end{tabular}

21 So zum Beispiel im Sammelband von Angelika Vetter, Erfolgsbedingungen lokaler Bürgerbeteiligungen, Wiesbaden 2008, oder bei Tobias Escher, a.a.O. (Fn. 18). 
Die Inanspruchnahme internetgestützter Beteiligungsvarianten ist bei den Einreichern herkömmlicher Petitionen teilweise erstaunlich hoch. 51,3 Prozent derjenigen, die eine herkömmliche Petition eingereicht haben und schon einmal mit Medien Kontakt aufgenommen haben, um ihre eigene Meinung zum Ausdruck zu bringen, haben dies auch schon mindestens einmal über das Internet gemacht. Dieses Ergebnis ist konsistent zu dem im vorherigen Abschnitt festgestellten allgemein hohen Niveau der Internetnutzung auch der Einreicher herkömmlicher Petitionen. Es wird aber auch deutlich, dass die internetaffinen Einreicher öffentlicher Petitionen für andere Formen der politischen Beteiligung nicht nur das Internet einsetzen, sondern Petitionen, Unterschriftensammlungen, Kontakte zu Politikern, Behörden und Medien durchaus auch in einem beträchtlichen Umfang (wenn auch immer mit einem Anteil unter 50 Prozent) über andere Medien (Brief, Telefon, Fax, persönliches Gespräch etc.) abwickeln.

\subsection{Inhalt und Absichten der eigenen Petition}

In Politik und Öffentlichkeit wird immer wieder diskutiert, ob das Petitionswesen in erster Linie den privaten Anliegen der Bürger dienen sollte oder in gleicher oder noch stärkerer Weise den politisch motivierten Bitten zu Gesetzesänderungen. ${ }^{22}$ Die Befragung zeigt nun, wie die Einreicher traditioneller und öffentlicher Petitionen selbst diese charakterisieren. ${ }^{23}$ Einer von fünf befragten Einreichern einer traditionellen Petition (21,3 Prozent) erklärte, dass sein Anliegen eine einzelne Person betrifft. Für die überwiegende Mehrheit (78,7 Prozent) bezieht sich der Inhalt der Petition auf die gesamte Bevölkerung oder Teile davon. Bei den Einreichern öffentlicher Petitionen kommt der Inhalt „einzelne Person“ mit 0,3 Prozent faktisch gar nicht vor, was allerdings mit der vorgegebenen Anforderung an öffentliche Petitionen zusammenhängt: Die schon erwähnte „Richtlinie“ des Bundestages zu den öffentlichen Petitionen sagt nicht nur aus, dass diese ein allgemeines Interesse zum Gegenstand haben müssen, sondern fordert des Weiteren, dass sich das Anliegen nicht erkennbar auf Personen beziehen darf. Tatsächlich haben sich die Petenten weitgehend an diese Vorgabe gehalten, denn kaum eine Eingabe wurde aus diesem Grund als öffentliche Petition abgelehnt (siehe unten).

Die Änderung und Verabschiedung von Gesetzen ist eine der zentralen Aufgaben des Bundestages, für die weitgehende Transparenz der Gegenstände und des Verfahrens selbstverständlich ist. Petitionen sind ein Mittel für die Bürger, auf diesen Prozess durch eigene Vorschläge einzuwirken. Ganz unabhängig davon, wie die Erfolgschancen von Petitionen für die Mitgestaltung von Gesetzesvorhaben einzuschätzen sind, zeigt die Befragung, dass im Bewusstsein der Petenten dieses Ziel sehr präsent ist und die große Mehrheit sowohl der

22 Vgl. etwa Stephan Löwenstein, a.a.O. (Fn. 19), S. 4. Zur Frage des politischen Charakters von Petitionen vgl. auch Ralf Lindner / Ulrich Riehm, a.a.O. (Fn. 6). Siehe auch den Beitrag von Ulrich Riehm, Christopher Coenen und Ralf Lindner in diesem Heft.

23 Ein bedeutender Teil des Fragebogens bezog sich auf das eigene Petitionsverfahren. Bei den Einreichern herkömmlicher Petitionen war dies die Petition, die der Befragte vor wenigen Tagen oder Wochen beim Bundestag eingereicht hatte und zu der er nun ein erstes Bestätigungsschreiben durch den Ausschussdienst erhielt, dem der Fragebogen beigelegt war. Bei den Einreichern öffentlicher Petitionen bezogen sich diese Verfahrensfragen auf die letzte Petition, die der Befragte als öffentliche Petition im Zeitraum September 2005 bis Dezember 2006 eingereicht hatte. 
Einreicher traditioneller (84,5 Prozent) als auch öffentlicher (93,0 Prozent) Petitionen eine Gesetzesänderung mit ihrer Petition anregen will. Diese beziehen sich, wie zu erwarten, ganz überwiegend auf Teile der Bevölkerung oder die gesamte Bevölkerung.

Bei der festgestellten Dominanz für nicht persönliche Inhalte und der Absicht, Gesetzesänderungen anzuregen, verwundert es nicht, dass die Befragten auch ein starkes Interesse an der Öffentlichkeit ihrer Petition formulieren (siehe Tabelle 6). ${ }^{24}$ Dies gilt sogar für die Einreicher traditioneller Petitionen.

\begin{tabular}{|c|c|c|}
\hline & \begin{tabular}{|c}
$\begin{array}{c}\text { Einreicher traditioneller } \\
\text { Petitionen }\end{array}$ \\
\end{tabular} & $\begin{array}{c}\text { Einreicher öffentlicher } \\
\text { Petitionen }\end{array}$ \\
\hline $\begin{array}{l}\text {... bei den zuständigen Stellen in Politik und } \\
\text { Verwaltung bekannt werden. }(\mathrm{n}=534,342)\end{array}$ & $97,4(89,0)$ & $99,1(91,2)$ \\
\hline $\begin{array}{l}\ldots \text { in der Öffentlichkeit und den Medien bekannt } \\
\text { werden. }(\mathrm{n}=483,330)\end{array}$ & $80,5(64,8)$ & $93,0(73,3)$ \\
\hline \multicolumn{3}{|c|}{$\begin{array}{l}\text { Frage: Bitte geben Sie an, wie zutreffend Sie die folgenden Aussagen finden. Meine Petition soll ... } \\
\text { Vierstufige Skale von „trifft voll und ganz zu“ bis "trifft gar nicht zu“. In der Tabelle sind die kumulier- } \\
\text { ten Anteile in Prozent für „trifft voll und ganz zu“ und „trifft eher zu“, in Klammern die Anteile für } \\
\text { "trifft voll und ganz zu“, angegeben. } \\
\text { Quelle: Eigene Erhebung } 2007 \text {. }\end{array}$} \\
\hline
\end{tabular}

Dieses Interesse an öffentlicher Diskussion und Aufmerksamkeit der eigenen Petition kommt auch im folgenden Fragenkomplex zum Ausdruck, der nur den Einreichern öffentlicher Petitionen vorgelegt wurde (siehe Tabelle 7). Die größte Hoffnung richtet sich für fast 90 Prozent der Befragten - wenig überraschend - darauf, dass sie mit einer öffentlichen Petition im Bundestag eine stärkere Berücksichtigung erfahren. ${ }^{25}$ Fast ähnlich hoch bewertet, aber vielleicht in einem eher instrumentellen Verhältnis zu diesem übergeordneten Ziel, stimmt die überwiegende Mehrheit der Aussage zu, dass sie ihr Anliegen öffentlich diskutieren wollen (83 Prozent). 80,8 Prozent erhoffen sich, über die öffentliche Petition mehr Unterstützer zu gewinnen und 72,5 Prozent, die größte Aufmerksamkeit zu erzielen.

Fasst man den Themenkomplex zu den Inhalten der eingereichten Petition und den damit verfolgen Absichten zusammen, kann die Auffassung, bei Petitionen an den Bundestag ginge es überwiegend um eine individuelle Beschwerde oder gar um einen persönlichen Härtefall, für den eine vertrauliche Behandlung gewünscht wird, nicht aufrechterhalten werden. Die große Mehrheit auch der Einreicher traditioneller Petitionen vertritt ein Anliegen, das sich nicht nur auf eine einzelne Person richtet, sondern auf eine Personengruppe oder die gesamte Gesellschaft, mit dem eine Gesetzesänderung angeregt wird und für das ein Interesse an einer Veröffentlichung besteht. Dies entspricht den Kriterien, die für die Zulassung einer öffentlichen Petition gelten, und zeigt, dass mit der Einführung öffentlicher Petitionen ein tatsächlich vorhandener Bedarf bei den Petenten aufgegriffen wurde.

24 Zum Komplex Öffentlichkeit von Petitionen vgl. Ulrich Riehm / Christopher Coenen / Ralf Lindner, a.a.O. (Fn. 22).

25 Faktisch mag dies im Einzelfall tatsächlich so sein, zum Beispiel wenn es gelingt, viele Unterstützer für eine Petition zu sammeln und die Medien auf eine solche Petition aufmerksam werden. Damit steht der Bundestag allerdings in der Gefahr, das Postulat aufzugeben, dass jede Petition gleich behandelt wird. 


\begin{tabular}{|c|c|}
\hline & $\begin{array}{l}\text { Einreicher öffentlicher } \\
\text { Petitionen }\end{array}$ \\
\hline $\begin{array}{l}\text {... eine stärkere Berücksichtigung meines Anliegens beim Bundestag } \\
\text { erhoffe. }(\mathrm{n}=335)\end{array}$ & $89,6(71,3)$ \\
\hline ... mein Anliegen öffentlich diskutieren kann. $(\mathrm{n}=311)$ & $83,0(50,5)$ \\
\hline ... die meisten Unterstützer/innen gewinnen kann. $(\mathrm{n}=313)$ & $80,8(53,7)$ \\
\hline ... die größte Aufmerksamkeit erzielen kann. $(\mathrm{n}=316)$ & $72,5(44,0)$ \\
\hline \multicolumn{2}{|c|}{$\begin{array}{l}\text { Frage: Bitte geben Sie an, wie zutreffend Sie die folgenden Aussagen finden. Ich habe mein Anliegen als } \\
\text { öffentliche Petition eingereicht, weil ich auf diese Weise ... } \\
\text { Vierstufige Skale von „trifft voll und ganz zu“ bis „trifft gar nicht zu“. In der Tabelle sind die kumulier- } \\
\text { ten Anteile in Prozent für „trifft voll und ganz zu“ und „trifft eher zu“, in Klammern die Anteile für } \\
\text { „trifft voll und ganz zu“, angegeben. } \\
\text { Quelle: Eigene Erhebungen } 2007 \text {. }\end{array}$} \\
\hline
\end{tabular}

Berücksichtigt man weiterhin die relative Unbekanntheit des Modellversuchs Öffentliche Petitionen, so scheint das Potenzial für öffentliche Petitionen viel größer, als die bisherigen Einreichungs- und Zulassungszahlen vermuten lassen.

\subsection{Zulassung der eigenen Eingabe als öffentliche Petition}

Die Befragung der Einreicher herkömmlicher Petitionen war gekoppelt an die Eingangsbestätigung durch den Ausschussdienst des Bundestages. Dabei wurde danach gefragt, was diese Eingangsbestätigung enthielt. Danach wurde den Petenten in der großen Mehrzahl (93,9 Prozent) eine weitere Prüfung ihres Anliegens zugesagt. Der häufigste Grund dafür, dass die Petition nicht weiter bearbeitet wurde, war die Nichtzuständigkeit des Bundestages mit 51,5 Prozent der zurückgewiesenen Petitionen $(n=33)$. Für je 15,2 Prozent war für die Nichtweiterbehandlung ausschlaggebend, dass bereits eine sachgleiche Petition beim Bundestag vorlag oder dass die Form der Eingabe ungenügend war. Andere Gründe waren bei 18,2 Prozent ausschlaggebend. Die Rückmeldung des Bundestags über die Zurückweisung der eigenen Petition wurde recht zwiespältig beurteilt (siehe Tabelle 8). Zwar stimmten

\begin{tabular}{|l|c|c|}
\hline Tabelle 8: Beurteilung der Ablehnung der eigenen Petition (in Prozent) \\
\hline & $\begin{array}{c}\text { Einreicher traditioneller } \\
\text { Petitionen } \\
(\mathrm{n}=24,31,21)\end{array}$ & $\begin{array}{c}\text { Einreicher öffentlicher } \\
\text { Petitionen } \\
(\mathrm{n}=138,159,124)\end{array}$ \\
\hline Meine Petition wurde inhaltlich verstanden. & 70,8 & 65,4 \\
\hline Die Begründung ist nachvollziehbar. & 58,1 & 45,3 \\
\hline Meine Petition wurde ausgewogen beurteilt. & 38,1 & 37,1 \\
\hline $\begin{array}{l}\text { Frage: Wie beurteilen Sie die Begründung, warum Ihre Petition nicht weiter geprüft oder bearbeitet } \\
\text { wird? / Wie beurteilen Sie die Begründung, warum ihre Petition nicht (oder nicht als öffentliche) zuge- } \\
\text { lassen wurde? Bitte geben Sie an, wie zutreffend Sie die folgenden Aussagen finden. Bewertung auf einer } \\
\text { vierstufigen Skale. Angegeben sind die kumulierten Anteilswerte in Prozent für ,trifft voll und ganz zu“ } \\
\text { und ,trifft eher zu“. } \\
\text { Quelle: Eigene Erhebungen 2007. }\end{array}$ \\
\hline
\end{tabular}


70,8 Prozent der Aussage zu, dass die eigene Petition von den Mitarbeitern des Bundestages „inhaltlich verstanden“ wurde (,trifft voll und ganz zu“ und „trifft eher zu“), und 58,1 Prozent fanden die Begründung des Bundestages nachvollziehbar. Hingegen erkannten nur 38,1 Prozent darin eine ausgewogene Beurteilung.

Bei den Einreichern einer öffentlichen Petition geht es im ersten Schritt der Prüfung beim Bundestag darum, ob die Eingabe als öffentliche Petition zugelassen werden kann oder als „normale“ (das heißt traditionelle) behandelt werden soll. Nach der Befragung liegt die Zulassungsquote als öffentliche Petition bei 50,6 Prozent $(n=346){ }^{26}$ 26,6 Prozent wurden zwar nicht als öffentliche, aber als herkömmliche Petition und 22,8 Prozent überhaupt nicht weiter geprüft. Als Gründe für die Nichtzulassung als öffentliche Petition wurden dabei, nach den Angaben der Befragten, die folgenden Gründe angegeben ( $n=167)$ : Das Anliegen ist nicht von allgemeinem Interesse (8,4 Prozent); es ist für eine sachliche öffentliche Diskussion nicht geeignet (5,4 Prozent); der Petent ist bereits mit einer oder mehreren Petitionen auf den Internetseiten des Bundestages präsent (3,0 Prozent); die Petition enthält Hinweise auf einzelne Personen (0,6 Prozent); sie enthält Links auf andere Webseiten (0,0 Prozent) oder Werbung für kommerzielle Produkte (0,0 Prozent). Weiterhin wurden als Gründe für die Zurückweisung auch als herkömmliche Petition angegeben: Sachgleiche Petition liegt bereits vor (35,9 Prozent); der Bundestag ist nicht zuständig (15,6 Prozent); die Petition wäre offensichtlich erfolglos (8,4 Prozent); sie ist offensichtlich unsachlich (1,8 Prozent); das Anliegen ist nicht erkennbar (0,6 Prozent); andere Begründungen (15,0 Prozent); der Ablehnungsgrund wurde vom Petenten nicht mehr erinnert $(5,4$ Prozent).

Die Bewertung der Ablehnungsgründe durch die Befragten entspricht dem oben schon festgestellten Muster bei den Einreichern traditioneller Petitionen. Auch hier gibt eine etwas kleinere - Mehrheit an, dass das eigene Anliegen offenbar inhaltlich verstanden wurde (65,4 Prozent) (siehe Tabelle 8). Keine Mehrheit (45,3 Prozent) findet sich für die Aussage, dass die Begründung nachvollziehbar sei. Etwas über ein Drittel (37,1 Prozent) erkennt in der Benachrichtigung über die Zurückweisung der eingereichten öffentlichen Petition eine ausgewogene Beurteilung.

Dieser sich hier andeutende Eindruck, dass die Einreicher öffentlicher Petitionen mit dem Zulassungsverfahren tendenziell unzufriedener sind als die Einreicher herkömmlicher Petitionen verstärkt sich, wenn man die summarische Frage nach der Zufriedenheit über die bisherige Behandlung einbezieht (siehe Tabelle 9). Dabei muss allerdings berücksichtigt werden, dass ein direkter Vergleich zwischen beiden Gruppen insofern problematisch ist, als die Einreicher herkömmlicher Petitionen diese Einschätzung im Wesentlichen auf Basis des Eingangsbestätigungsschreibens - mit einer eventuellen Zurückweisung als Petition gegeben hatten, während es unter den Einreichern öffentlicher Petitionen solche gibt, bei

26 Nach den Angaben des Bundestages ist die Zulassungsquote öffentlicher Petitionen mit 37,3 Prozent im Jahr 2006 und 38,5 Prozent im Jahr 2007 deutlich niedriger. Unter den 698 für die Befragung angeschriebenen Einreichern öffentlicher Petitionen waren nach Angaben des Bundestages nur circa 300, deren Petition als öffentliche Petition zugelassen wurde. Das entspricht einer etwas höheren Zulassungsquote von 43,0 Prozent, die aber immer noch niedriger ist als die in der Befragung mit 50,6 Prozent. Man muss deshalb davon ausgehen, dass die Einreicher öffentlicher Petitionen, deren Petition nicht zugelassen wurde, weniger an der Befragung teilgenommen haben als diejenigen, deren Einreichung als öffentliche Petition akzeptiert wurde. 
denen das gesamte Verfahren bereits abgeschlossen war, bei allen aber mindestens drei Monate seit der Einreichung vergangen waren. Gerade deshalb sollte der hohe Anteil derjenigen, die mit der Behandlung im Rahmen des neuen Verfahrens der öffentlichen Petitionen nicht zufrieden waren (58,3 Prozent), nachdenklich machen. Es erscheint dabei durchaus plausibel, dass ein wichtiger Gesichtspunkt für diese kritische Haltung, die relativ hohe Rate der Nichtzulassung für die öffentlichen Petitionen ist. ${ }^{27}$

\begin{tabular}{|l|c|c|}
\hline Tabelle 9: Zufriedenheit mit bisheriger Petitionsbehandlung (in Prozent) \\
\hline & $\begin{array}{c}\text { Einreicher traditioneller } \\
\text { Petitionen }(\mathrm{n}=475)\end{array}$ & $\begin{array}{c}\text { Einreicher öffentlicher } \\
\text { Petitionen }(\mathrm{n}=329)\end{array}$ \\
\hline sehr zufrieden & 40,6 & 9,7 \\
\hline eher zufrieden & 35,8 & 31,9 \\
\hline eher nicht zufrieden & 13,3 & 34,3 \\
\hline gar nicht zufrieden & 10,3 & 24,0 \\
\hline $\begin{array}{l}\text { Frage: Wie zufrieden sind Sie bislang mit der Behandlung Ihrer Petition? } \\
\text { Quelle: Eigene Erhebungen 2007. }\end{array}$ \\
\hline
\end{tabular}

\subsection{Nutzung und Bewertung des Diskussionsforums}

Das Diskussionsforum, also die Möglichkeit, öffentlich im Internet über eine Petition Meinungen austauschen zu können, ist eines der innovativen Elemente des Modellversuchs. 93,5 Prozent der befragten Einreicher öffentlicher Petitionen $(n=338)$ fanden diese Funktion prinzipiell sehr wünschenswert, weitere 6,2 Prozent eher wünschenswert, womit eine fast 100-prozentige Zustimmungsrate erreicht ist. Ob diese Funktion aber auch von den Petenten genutzt und wie sie nach der eigenen Nutzungserfahrung bewertet wird, kann man auf Grundlage der Befragung nun besser einschätzen. Die Nutzung des Diskussionsforums erscheint mit einem Anteil von 32,0 Prozent „passiven“ (nur lesenden) und einem Anteil von 50,3 Prozent „aktiven“ (lesenden und schreibenden) Petenten außerordentlich gut. Man sollte sich dabei nochmals vor Augen halten, dass die Einreichung und Veröffentlichung einer Petition im Internet des Bundestags automatisch mit der Einrichtung eines Diskussionsforums verbunden ist, egal ob der Petent dies wünscht oder nicht. Es könnte also gut sein, dass bestimmte Petenten zum Beispiel nur an der Veröffentlichung ihrer Petition oder an der Mitzeichnungsfunktion interessiert sind, nicht aber am Diskussionsforum. Dieser Anteil der Nichtnutzer des Diskussionsforums ist mit 17,7 Prozent relativ gering. Hervorzuheben ist auch, dass sich die Hälfte der Petenten nach eigenen Angaben aktiv schreibend am Diskussionsforum beteiligt, was im Vergleich mit Erfahrungen aus anderen Kommunikations- und Diskussionsmedien (E-Mail-Listen, Blogs etc.) außergewöhnlich hoch erscheint.

27 Befragungen in England zur Zufriedenheit mit der Behandlung von Beschwerden an lokale Behörden und die Bevölkerungsbefragung zum Petitionswesen in Deutschland zeigen allerdings ebenfalls nur eine Zufriedenheitsrate von etwa 30 Prozent. Vgl. Ulrich Riehm, Vorwort zu: Barbara Lippa / Herbert Kubicek / Stehphan Bröchler, a.a.O. (Fn. 2), S. 6. 


\begin{tabular}{|l|c|}
\hline \multicolumn{2}{|l|}{ Tabelle 10: Bewertung des Diskussionsforums (in Prozent) } \\
\hline & $\begin{array}{c}\text { Einreicher öffentlicher } \\
\text { Petitionen }\end{array}$ \\
\hline Das Diskussionsforum war leicht zu finden. (n = 140) & 82,1 \\
\hline Das Diskussionsforum war übersichtlich und leicht zu handhaben. ( $\mathrm{n}=141)$ & 74,5 \\
\hline Die Moderation der Diskussion war neutral. ( $\mathrm{n}=102)$ & 79,4 \\
\hline Die Moderation der Diskussion war hilfreich. $(\mathrm{n}=90)$ & 66,7 \\
\hline Die Diskussion im Forum verlief sachlich und ohne Beleidigungen. $(\mathrm{n}=136)$ & 76,5 \\
\hline Die Diskussion im Forum war sehr informativ. (n =138) & 67,4 \\
\hline $\begin{array}{l}\text { Frage: Bitte teilen Sie uns mit, inwiefern Sie die folgenden Aussagen zutreffend finden. Angegeben sind } \\
\text { die kumulierten Anteile in Prozent für ,trifft voll und ganz zu“ und „trifft eher zu“. Befragt wurden nur }\end{array}$ \\
$\begin{array}{l}\text { Einreicher öffentlicher Petitionen, deren Petition als öffentliche Petition zugelassen wurde, und die das } \\
\text { Diskussionsforum lesend oder auch schreibend genutzt haben. } \\
\text { Quelle: Eigene Erhebungen 2007. }\end{array}$ \\
\hline
\end{tabular}

Die Petenten, die das Diskussionsforum selbst genutzt haben, sind damit überwiegend zufrieden (siehe Tabelle 10). In der Befragung wurden drei Aspekte unterschieden: die Gebrauchstauglichkeit des Forums, die Moderation durch den Bundestag und die Inhalte der Diskussion. Drei von vier Befragten stimmten der Aussage zu, dass das Diskussionsforum übersichtlich und leicht zu handhaben sei. Vier von fünf bescheinigten der Moderation Neutralität und zwei von drei eine hilfreiche Funktion. ${ }^{28}$ Ähnlich hoch ist die inhaltliche Bewertung der Diskussionen. Etwas über zwei Drittel der Befragten stimmten der Aussage voll und ganz beziehungsweise eher zu, dass die Diskussion im Forum sehr informativ war. $^{29}$

\subsection{Bewertung des Modellversuchs Öffentliche Petitionen}

Beide befragten Petentengruppen wurden - unabhängig von einer konkreten Erfahrung mit öffentlichen Petitionen - nach ihren allgemeinen Einschätzungen zu den Merkmalen des Modellversuchs Öffentliche Petitionen befragt. Es zeigt sich dabei eine im Großen und Ganzen hohe positive Zustimmung und ein besonders starkes Interesse an der Öffentlichkeit der Petitionen (siehe Tabelle 11), wie dies oben bereits für die selbst eingereichte Petition festgestellt wurde. Die Einreicher herkömmlicher Petitionen finden die Möglichkeit zur Veröffentlichung zu 90,5 Prozent, die Mitzeichnungsmöglichkeit im Internet zu 87,4 Prozent und die Diskussion im Internetforum zu 85,7 Prozent eher oder sehr interessant. Noch positiver äußern sich die Einreicher öffentlicher Petitionen zu den Komponenten des Modellversuchs: Die Veröffentlichung der Petition finden 99,7 Prozent, die Mitzeichnung 98,2 Prozent und die Forumsdiskussion 95,8 Prozent eher oder sehr wünschenswert.

28 Allerdings äußern bei den Fragen zur Moderation mehr als sonst „keine Meinung“, was an einer geringen Präsenz der Moderatoren in den Foren liegen könnte.

29 Die abgeschwächte Zustimmung „trifft eher zu“ mit 52,9 Prozent überwiegt dabei eindeutig vor der vorbehaltlosen Zustimmung ,trifft voll und ganz zu“ mit nur 14,5 Prozent. 


\begin{tabular}{|c|c|c|}
\hline \multicolumn{3}{|c|}{ Tabelle 11: Bewertung der Merkmale öffentlicher Petitionen (in Prozent) } \\
\hline & $\begin{array}{c}\text { Einreicher traditioneller } \\
\text { Petitionen }\end{array}$ & $\begin{array}{c}\text { Einreicher öffentlicher } \\
\text { Petitionen }\end{array}$ \\
\hline $\begin{array}{l}\text {... werden auf den Internetseiten des Bundestages } \\
\text { veröffentlicht. }(\mathrm{n}=472,338)\end{array}$ & $90,5(59,5)$ & $99,7(93,5)$ \\
\hline $\begin{array}{l}\text {... können auf den Internetseiten des Bundestages } \\
\text { von Unterstützern/-innen unterschrieben werden } \\
\text { (Mitzeichnung). }(\mathrm{n}=452,340)\end{array}$ & $87,4(65,0)$ & $98,2(91,5)$ \\
\hline $\begin{array}{l}\ldots \text { können auf den Internetseiten des Bundestages } \\
\text { in einem öffentlichen Forum diskutiert werden. } \\
(\mathrm{n}=462,337)\end{array}$ & $85,7(54,8)$ & $95,8(77,2)$ \\
\hline $\begin{array}{l}\text {... können auf öffentlichen Sitzungen im Petitions- } \\
\text { ausschuss des Bundestages beraten werden. ( } \mathrm{n}= \\
508,338)\end{array}$ & $95,1(71,7)$ & $97,6(84,9)$ \\
\hline \multicolumn{3}{|c|}{$\begin{array}{l}\text { Frage: An die Einreicher traditioneller Petitionen: Wie interessant finden Sie die folgenden Merkmale } \\
\text { von öffentlichen Petitionen? Öffentliche Petitionen ... } \\
\text { An die Einreicher öffentlicher Petitionen: Was halten Sie grundsätzlich davon, dass öffentliche Petitio- } \\
\text { nen ... } \\
\text { Vierstufige Skale von „trifft voll und ganz zu“ bis „trifft gar nicht zu“. In der Tabelle sind angegeben die } \\
\text { kumulierten Anteile in Prozent für "trifft voll und ganz zu“ und „trifft eher zu“, in Klammern die Antei- } \\
\text { le für „trifft voll und ganz zu“. } \\
\text { Quelle: Eigene Erhebungen } 2007 .\end{array}$} \\
\hline
\end{tabular}

Die Forumskomponente zeichnet den Modellversuch besonders aus. Es wird damit signalisiert, dass es nicht nur um einen anderen medialen Einreichungsweg für Petitionen geht, auch nicht allein um eine „Unterschriftensammelstelle“, sondern dass für die Beurteilung von politischen Sachverhalten der Austausch von Argumenten notwendigerweise dazugehört. Dies drückt sich auch in sehr hohen Erwartungen an die Funktion des Diskussionsforums bei den Einreichern öffentlicher Petitionen aus: 78 Prozent sehen den Zweck des Diskussionsforums darin, dass sich Bürger eine Meinung zur jeweiligen Petition bilden können; für 65 Prozent liegt der Zweck in der inhaltlichen Unterstützung des Petitionsausschusses bei der Beurteilung der jeweiligen Petition; 57 Prozent sehen im Diskussionsforum die Möglichkeit für einen Austausch zwischen den Bürgern; 52 Prozent sind der Auffassung, dass das Forum dazu dienen sollte, Kontakt zwischen Abgeordneten und Bürgern herzustellen.

Damit wird die doppelte Funktion für die Mehrheit der Einreicher öffentlicher Petitionen deutlich: Mit dem Diskussionsforum richtet man sich sowohl an die Öffentlichkeit als auch an den Bundestag. Dass die zweite Erwartung weitgehend nicht erfüllt wird, da gegenwärtig weder Abgeordnete auf den Diskussionsforen mitdiskutieren noch diese systematisch ausgewertet und die Auswertungen dem Petitionsausschuss als Beratungsgrundlage zugestellt werden, steht auf einem anderen Blatt. ${ }^{30}$

30 Ulrich Riehm / Christopher Coenen / RalfLindner / Clemens Blümel, a.a.O. (Fn. 6), S. 233 - 235, S. $250-253$. 


\section{3. Öffentliche Petitionen: objektiver Bedarf und große Zustimmung}

Der Bundestag hat mit dem Modellversuch Öffentliche Petitionen und seiner Fortführung einen bedeutenden Modernisierungsschritt unternommen, der weithin Zustimmung und Anerkennung gefunden hat. ${ }^{31}$ Nicht nur wurde das Internet ins Petitionswesen eingeführt, sondern auch die Aspekte der Öffentlichkeit und Diskursivität im Petitionsverfahren wurden gestärkt. In der begleitenden Studie des TAB zum Modellversuch wurden zum ersten Mal Petenten nach ihren persönlichen Merkmalen, nach ihren Petitionen, nach der Bewertung des erlebten Verfahrens und nach den Merkmalen des Modellversuchs befragt.

Fasst man die Ergebnisse zusammen, ist es bislang nur sehr begrenzt gelungen, über das Internet und das Instrument der öffentlichen Petitionen neue Bevölkerungsgruppen zu erreichen. Die Einreicher öffentlicher Petitionen sind zwar deutlich jünger als die Einreicher herkömmlicher Petitionen, die starke Männerdominanz und das deutliche Übergewicht von Petenten mit Hochschulbildung - Indizien sozialer Ungleichheit - wurde jedoch nicht reduziert. Bezieht man das Niveau politischer Aktivität mit ein, verstärkt sich dieser Eindruck weiter: Es sind nicht die politisch Inaktiven, die durch Petitionen und öffentliche Petitionen besonders angesprochen werden, sondern die ohnehin politisch Engagierten. Allerdings ist zu berücksichtigen, dass die Befragungen bereits nach einem guten Jahr des Modellversuchs durchgeführt wurden. Zukünftige Untersuchungen wären notwendig, um zu klären, ob die bessere Nutzbarkeit öffentlicher Petitionen, die Steigerung ihrer Bekanntheit und weitere Faktoren die Inanspruchnahme durch breitere Bevölkerungsschichten verbessern.

Dennoch kann man feststellen, dass die öffentlichen Petitionen nicht nur auf eine extrem große Zustimmung gestoßen sind, sondern auch einen objektiv vorhandenen Bedarf ansprechen, denn die Einreicher herkömmlicher Petitionen sehen den Inhalt ihrer Petition überwiegend im Bereich der „Bitten zur Gesetzgebung“ und formulieren konsequenterweise ein großes Interesse an der Öffentlichkeit ihrer Petition und den durch den Modellversuch eingeführten diskursiven Elementen. Es ist deshalb zu vermuten, dass das Potenzial für öffentliche Petitionen mit gegenwärtig rund zwei Prozent unter allen Petitionen längst nicht ausgeschöpft ist.

Welche Konsequenzen aus diesen beiden zentralen Erkenntnissen zu ziehen wären, kann hier nur angedeutet werden. Will man die bisher unterrepräsentierten Bevölkerungsgruppen näher an das Petitionswesen heranführen, muss es nicht nur besser bekannt gemacht, sondern auch bestimmte Hürden reduziert werden. Dies gilt vermutlich besonders für das Schriftlichkeitspostulat der Einreichung. Immerhin 27 Prozent der Bevölkerung würden eine persönliche Vorsprache bei einer Eingabestelle einer schriftlichen - postalischen oder elektronischen - Eingabe vorziehen. ${ }^{32}$ Auf die bisher geringe Ausschöpfung des Potenzials öffentlicher Petitionen könnte man auch so reagieren, dass öffentliche Petitionen zum Regelfall erklärt werden - was den mehrheitlichen Wünschen der Petenten entsprechen würde - ohne damit vertraulich zu behandelnde Petitionen auszuschließen. ${ }^{33}$

31 Zum Beispiel wurde der Petitionsausschuss am 4. Dezember 2008 mit dem „Politik-Award“ der Zeitschrift für Politik \& Kommunikation in der Kategorie „Innovation“ ausgezeichnet.

32 Barbara Lippa / Herbert Kubicek / Stephan Bröchler, a.a.O. (Fn. 2). Vgl. zu einer „Multikanalstrategie" im Petitionswesen auch Ulrich Riehm / Christopher Coenen / RalfLindner / Clemens Blümel, a.a.O. (Fn. 6), S. $258-259$.

33 Vgl. Ulrich Riehm / Christopher Coenen / RalfLindner, a.a.O. (Fn. 22). 